\title{
Antibacterial activity and dentin bonding ability of combined use of Clearfil SE Protect and sodium hypochlorite
}

\author{
Ilijana MURATOVSKA ${ }^{1}$, Haruaki KITAGAWA², Nanako HIROSE³ ${ }^{3}$ Ranna KITAGAWA ${ }^{3}$ and Satoshi IMAZATO² \\ ${ }^{1}$ Department of Cariology and Endodontics, Faculty of Dental Medicine, University Ss. Cyril and Methodius, Vodnjanska 17, 1000 Skopje, \\ Macedonia \\ ${ }^{2}$ Department of Biomaterials Science, Osaka University Graduate School of Dentistry, 1-8 Yamadaoka, Suita, Osaka 565-0871, Japan \\ ${ }^{3}$ Department of Restorative Dentistry and Endodontology, Osaka University Graduate School of Dentistry, 1-8 Yamadaoka, Suita, Osaka 565-0871, \\ Japan \\ Corresponding author, Haruaki KITAGAWA; E-mail: h-kita@dent.osaka-u.ac.jp
}

The aim of this study was to evaluate the antibacterial activity and dentin bonding ability of a commercial self-etch adhesive Clearfil SE Protect (Kuraray Noritake Dental, Tokyo, Japan) in combination with sodium hypochlorite (NaOCl). Agar disc diffusion tests and measurement of minimum inhibitory/bactericidal concentrations (MIC/MBC) against Streptococcus mutans were performed to evaluate antibacterial effects. The mixture solution of $5.25 \% \mathrm{NaOCl}$ and the primer of Clearfil SE Protect demonstrated less antibacterial activity than primer only. In microtensile bond strength tests using non-carious human molars, pretreatment with $5.25 \% \mathrm{NaOCl}$ aqueous solution had no influence on the bond strength of Clearfil SE Protect. These results indicate that pretreatment with $\mathrm{NaOCl}$ does not influence the bonding ability of Clearfil SE Protect, while their combined use does not enhance cavity disinfecting effects.

Keywords: Adhesive, Disinfection, Quaternary ammonium compound, $\mathrm{NaOCl}$

\section{INTRODUCTION}

Caries is treated by surgical removal of the infected tooth structure, however, excavation frequently results in a complex substrate involving areas of caries-infected, caries-affected, sclerotic, and eroded dentin ${ }^{1}$. Incomplete removal of infected dentin is sometimes recommended, especially in clinical situations of deep carious lesions, to preserve pulp tissue vitality ${ }^{2}$. Therefore, cavity disinfection to reduce residual bacteria is essential for the successful treatment of caries, and attempts to create restorative materials possessing antibacterial effects remains an important challenge ${ }^{3}$.

Clearfil SE Protect (Kuraray Noritake Dental, Tokyo, Japan) is an antibacterial self-etch adhesive system. It contains an antibacterial monomer 12-methacryloyloxy dodecylpyridinium bromide (MDPB) in its primer, which is synthesized by combining a methacryloyl group with a quaternary ammonium compound (QAC) ${ }^{4)}$. MDPB as a QAC is proposed to interact with negatively charged bacterial cell surfaces, causing a disturbance to the charge balance and damage to the cell membrane ${ }^{5}$. Because of its rapid bactericidal activity against cariogenic bacteria at the unpolymerized stage, MDPB has been incorporated into the self-etching primer of Clearfil SE Protect to provide cavity disinfecting effects. In addition, the ability of MDPB to polymerize is advantageous because it does not influence the bonding ability of adhesives ${ }^{6}$.

Sodium hypochlorite $(\mathrm{NaOCl})$ is frequently used for disinfection of the tooth surface. Several studies have reported the role of $\mathrm{NaOCl}$ used as a disinfectant prior to the bonding procedure ${ }^{7,8)}$. It is considered that the mechanism for the germicidal activity of $\mathrm{NaOCl}$ is the inhibition of enzyme activity essential for bacterial growth, damage to the membrane and DNA, and perhaps injury to the membrane transport capacity ${ }^{9}$. Thus, the mechanism underlying antibacterial actions of $\mathrm{NaOCl}$ differs from that of MDPB. Therefore, combined use of $\mathrm{NaOCl}$ with Clearfil SE Protect may provide additional effects in terms of antibacterial activity. Conversely, Ozturk and $\mathrm{Ozer}^{10)}$ reported that $\mathrm{NaOCl}$ application decreased the bond strength of Clearfil SE Bond (Kuraray Noritake Dental) to dentin. Because Clearfil SE Protect has similar components to Clearfil SE Bond except for the inclusion of MDPB in the primer and encapsulated sodium fluoride particles in the bonding resin, it is important to assess the influence of $\mathrm{NaOCl}$ pretreatment on the bonding ability of Clearfil SE Protect.

In this study, to examine the first null hypothesis that the use of $\mathrm{NaOCl}$ provides additional antibacterial effects to Clearfil SE Protect primer, antibacterial activity was evaluated by the agar disc diffusion test and measurement of minimum inhibitory/bactericidal concentrations (MIC/MBC) against Streptococcus mutans. The second null hypothesis tested was that pretreatment with $\mathrm{NaOCl}$ does not reduce the bonding ability of Clearfil SE Protect, which was measured by the microtensile bond strength test.

\section{MATERIALS AND METHODS}

Agar disc diffusion tests

The materials used for agar disc diffusion tests included a commercial two-step self-etch adhesive (Clearfil SE Protect primer, Kuraray Noritake Dental) and a 
commercial $10 \% \mathrm{NaOCl}$ solution (Neo Cleaner, Neo Dental Chemical Products, Tokyo, Japan). These solutions were mixed or diluted with water, and four test solutions were prepared as follows:

Group 1: Clearfil SE Protect primer (Primer)

Group 2: $5.25 \% \mathrm{NaOCl}$ solution $(\mathrm{NaOCl})$

Group 3: Clearfil SE Protect primer+5.25\% NaOCl solution with 1:1 vol ratio (Primer/ $\mathrm{NaOCl})$

Group 4: Clearfil SE Protect primer+distilled water with 1:1 vol ratio (Primer/Water).

Agar disc diffusion tests were performed as previously reported ${ }^{11)}$. Briefly, $S$. mutans strain NCTC10449 was used and cultivated in Brain Heart Infusion (BHI; Becton Dickinson, Sparks, MD, USA) broth at $37^{\circ} \mathrm{C}$. After anaerobic incubation for $24 \mathrm{~h}$, $300 \mu \mathrm{L}$ of bacterial suspension was spread onto a BHI agar plate. A 20- $\mu \mathrm{L}$ aliquot of each solution of the four test groups was impregnated into a sterile paper disc (diameter: $6 \mathrm{~mm}$, thickness: $1.5 \mathrm{~mm}$ ) and placed on an agar plate inoculated with the bacterial suspension. A $20-\mu \mathrm{L}$ volume was chosen as the optimum volume for impregnation into a paper disc without overflow of the test solution. Plates were incubated anaerobically for $48 \mathrm{~h}$ at $37^{\circ} \mathrm{C}$, and the diameter of the inhibition halo was measured at three points using a caliper (Mitutoyo, Tokyo, Japan). The sizes of the inhibition zones were calculated by the following equation:

Size of inhibition zone $=(I-D) / 2$

where $I=$ mean of three measurements of the diameter of inhibition halo $(\mathrm{mm})$ and $D=$ diameter of the paper disc $(6 \mathrm{~mm})$. The tests were repeated three times.

\section{MIC and MBC measurements}

$\mathrm{MIC}$ and $\mathrm{MBC}$ of Primer, $\mathrm{NaOCl}$, and Primer $/ \mathrm{NaOCl}$ as prepared above against $S$. mutans were determined by serial microdilution assay as previously reported ${ }^{11}$. Serial two-fold dilutions of each disinfectant were made in wells of a 96-well microplate containing BHI broth, and test samples $(50 \mu \mathrm{L}$; concentrations ranged from 0.000095 to $25 \%$ of the original solution) were prepared. S. mutans suspension $(50 \mu \mathrm{L})$ at approximately $2 \times 10^{6}$ colony-forming units/mL was inoculated into each well. The plates were then incubated anaerobically at $37^{\circ} \mathrm{C}$ for $48 \mathrm{~h}$. The MIC value was determined as the lowest concentration in the well at which turbidity was not observed by visual examination. Suspensions from wells that showed no bacterial growth were inoculated onto BHI agar plates. After subculture for $48 \mathrm{~h}$, the MBC value was determined as the lowest concentration that showed no colony formation on the plates. The tests were repeated five times.

\section{Microtensile bond strength tests}

Twenty non-carious human molars were randomly divided into two groups (described below) to test the treatment of dentin surfaces. The occlusal enamel of the crown was removed using a low-speed diamond saw (Isomet 2000, Buehler, Lake Bluff, IL, USA) and the dentin surface was polished with 600 -grit silicon carbide paper. To eliminate the influence of differences among teeth, one tooth specimen was divided into two pieces and one piece from each tooth was allocated to each treatment group.

In the first group, control (SEP), the dentin surface was treated with Clearfil SE Protect (Kuraray Noritake Dental). Briefly, the primer of Clearfil SE Protect was applied to the dentin surface for $20 \mathrm{~s}$ using a sponge supplied by the manufacturer. After the surface was dried using a gentle air blower, the bonding resin of Clearfil SE Protect was applied and light-cured for 10 $\mathrm{s}$ using a $1,000 \mathrm{~mW} / \mathrm{cm}^{2}$ LED curing device (PenCure, Morita, Kyoto, Japan). In the second group, the dentin surface was treated with $5.25 \% \mathrm{NaOCl}$ solution using a cotton pellet for $10 \mathrm{~s}$, washed with water for $10 \mathrm{~s}$, and then dried before application of Clearfil SE Protect.

A resin composite (Clearfil AP-X, Shade A3, Kuraray Noritake Dental) was built up in three layers to a height of $5 \mathrm{~mm}$. Light-curing was performed using the LED curing device (PenCure) for $20 \mathrm{~s}$ for each layer, and the specimens were immediately placed in distilled water. After storage at $37^{\circ} \mathrm{C}$ for $24 \mathrm{~h}$, the bonded specimens were clamped into the adopted holder and sectioned perpendicular to the bonding surface using the lowspeed diamond saw to obtain rectangular sticks $(1 \times 1$ $\mathrm{mm}, 8-9$-mm long). Each specimen was attached to a jig with an adhesive (Model Repair Pink, Dentsply Sankin, Tochigi, Japan) placed in a universal testing machine (EZ Test, Shimadzu, Kyoto, Japan) and the microtensile bond strength test was conducted at a crosshead speed of $1 \mathrm{~mm} / \mathrm{min}$. The cross-sectional area of each specimen was measured using a digital caliper (Absolute Digimatic Caliper, Mitutoyo, Kanagawa, Japan) and the bond strength value was calculated by dividing the load by the bonded area. The fracture mode was observed using a stereoscopic microscope (SMZ-U, Nikon, Tokyo, Japan) at $20 \times$ magnification to determine failure modes. Failure modes were classified as adhesive failure, cohesive failure within the tooth, or mixed failure (combination of adhesive failure and cohesive failure).

\section{Statistical analysis}

The results of agar disc diffusion tests were statistically analyzed using analysis of variance (ANOVA) and Student-Newman-Keuls post hoc test with a significance level of $p<0.05$ (SPSS Statistics 21, IBM-SPSS, Chicago, IL, USA). The results of microtensile bond strength tests were statistically analyzed using Student's $t$-test with a significance level of $p<0.05$.

\section{RESULTS}

\section{Agar disc diffusion tests}

The sizes of inhibition zones produced by agar disc diffusion tests are shown in Fig. 1. All test groups produced inhibition zones against $S$. mutans. A significantly smaller inhibition zone was observed for $\mathrm{NaOCl}$ compared with that for Primer $(p<0.05$, ANOVA, Student-Newman-Keuls post hoc test). The size of the inhibition zone for Primer/Water was approximately half of that for Primer. Although the mean size of the 


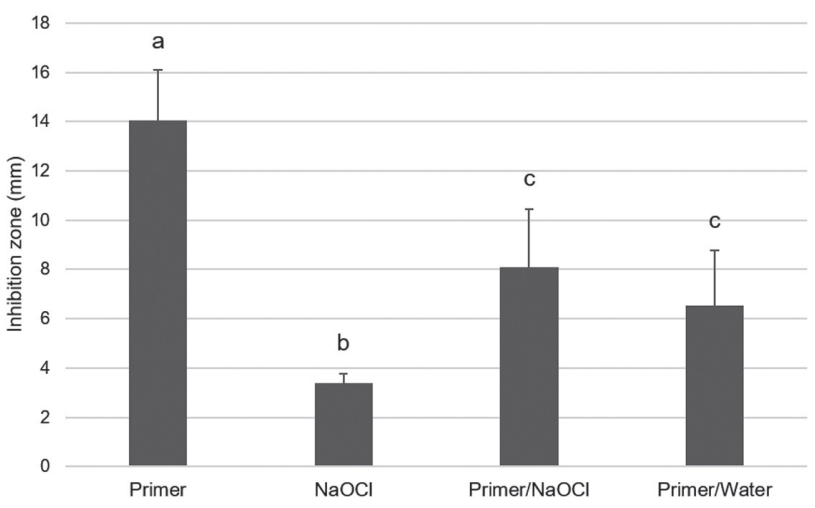

Fig. 1 Size of inhibition zones produced in agar disc diffusion tests.

The bar represents the standard deviation of three replicates. Groups that do not share the same superscript letter are significantly different $(p<0.05$, ANOVA, Student-Newman-Keuls post hoc test).

Table 1 MIC and $\mathrm{MBC}$ values of Primer, $\mathrm{NaOCl}$, and Primer/ $\mathrm{NaOCl}$ against $S$. mutans expressed as the percentage of the original solution (\%)

\begin{tabular}{lll}
\hline & MIC & MBC \\
\hline Primer & 0.012 & 0.098 \\
$\mathrm{NaOCl}$ & 12.5 & 12.5 \\
Primer $/ \mathrm{NaOCl}$ & 0.024 & 0.195 \\
\hline
\end{tabular}

inhibition zone for Primer/ $\mathrm{NaOCl}$ was greater than that for Primer/Water, no significant differences were observed between the two groups ( $p>0.05$, ANOVA, Student-Newman-Keuls post hoc test).

\section{$M I C$ and $M B C$ measurements}

Table 1 shows the MIC and MBC values against $S$. mutans expressed as the percentage of the original solution. The same results were obtained for five repetitions of the tests. $\mathrm{NaOCl}$ demonstrated much greater MIC and MBC values than Primer only. The $\mathrm{MIC}$ and $\mathrm{MBC}$ values of Primer/ $\mathrm{NaOCl}$ were two times greater than those of Primer only.

\section{Microtensile bond strength tests}

Microtensile bond strengths for SEP and $\mathrm{NaOCl}+\mathrm{SEP}$ are shown in Fig. 2, and failure modes are presented in Table 2. No significant differences in bond strength were observed between the two groups $(p>0.05$, Student's $t$-test). For SEP, adhesive failure was observed in all specimens. For $\mathrm{NaOCl}+\mathrm{SEP}$, adhesive failure was observed in $50 \%$ of the specimens, and cohesive or mixed failure was observed in 20 or $30 \%$ of the specimens, respectively.

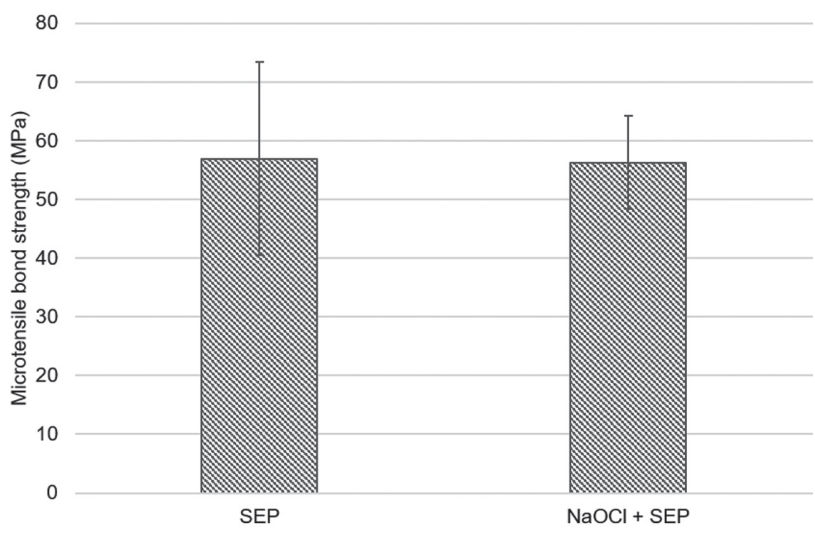

Fig. 2 Microtensile bond strength of Clearfil SE Protect to dentin with or without $\mathrm{NaOCl}$ pretreatment.

The bar represents the standard deviation of ten specimens. No significant differences between the two groups were observed ( $p>0.05$, Student's $t$-test).

Table 2 Results of failure mode analysis

\begin{tabular}{lcc}
\hline & SEP & NaOCl+SEP \\
\hline Adhesive & $10(100 \%)$ & $5(50 \%)$ \\
Cohesive & $0(0 \%)$ & $2(20 \%)$ \\
Mixed & $0(0 \%)$ & $3(30 \%)$ \\
\hline
\end{tabular}

\section{DISCUSSION}

To improve dental restoration failure rates, much attention has been paid to antibacterial effects of materials applied prior to direct filling materials. Cavity disinfectants are used to eradicate residual bacteria after surgical removal of the infected tooth or tooth preparation for direct/indirect restorative treatments. $\mathrm{NaOCl}$ is a well-known, nonspecific proteolytic agent as well as dissolver for magnesium and carbonate ions ${ }^{12}$. Therefore, $\mathrm{NaOCl}$ is used to remove the smear layer and collagen on dentin surfaces. The role of $\mathrm{NaOCl}$ used as a disinfectant on prepared dentin prior to the bonding procedure has been investigated ${ }^{13)}$. Due to its potent, Dijken demonstrated that acceptable bonded restorations were achieved after pretreatment of a tooth with $\mathrm{NaOCl}^{14)}$. Since then, the benefit of $\mathrm{NaOCl}$ against bacteria on dentin surfaces has also been demonstrated $^{15-17)}$.

QACs, such as cetylpyridinium chloride or benzalkonium chloride, are widely used as antibacterial agents ${ }^{18)}$. QACs are proposed to interact with negatively charged bacterial cell surfaces, causing a disturbance to the charge balance and damage to the cell membrane ${ }^{5)}$. In the $1990 \mathrm{~s}$, Imazato et al. developed the antibacterial monomer MDPB by combining a QAC with a methacryloyl group ${ }^{19)}$. As a derivative of $\mathrm{QAC}, \mathrm{MDPB}$ 
has strong antibacterial activity before polymerization. In addition, the antibacterial component is immobilized in a polymer network by polymerization of MDPB, and such immobilized antimicrobial does not leach out from cured resins. A number of studies have reported that MDPB has antibacterial activity against various dental caries-related bacteria ${ }^{20-22)}$. By employing an MDPBcontaining antibacterial, self-etching primer, the world's first adhesive system for restoration with antibacterial effects was successfully commercialized in 2004.

S. mutans is one of the major dental pathogens associated with the initiation of dental caries and also well recognized in residual and secondary caries. To evaluate the disinfectant ability of $\mathrm{NaOCl}$ in combination with Clearfil SE Protect primer containing MDPB, agar disc diffusion tests against $S$. mutans were conducted. $\mathrm{NaOCl}$ demonstrated a much smaller inhibition zone compared with Primer, indicating that the antibacterial activity of $\mathrm{NaOCl}$ was fundamentally weak. The size of the inhibition zone for Primer/Water was approximately half of that for Primer. This is because MDPB in the primer was diluted to half of its concentration due to mixture with water. In addition, no significant differences in size of inhibition zones were observed between Primer/NaOCl and Primer/Water. Thus, in agar disc diffusion tests, the additional effects of $\mathrm{NaOCl}$ to enhance antibacterial activity of Clearfil SE Protect primer were not observed.

It is not possible to precisely compare antibacterial activities by using agar disc diffusion tests because diffusivity of $\mathrm{NaOCl}$ in the agar might have been interrupted by the components of Clearfil SE Protect primer. Therefore, to compare intrinsic antibacterial activities, the MIC and MBC values of Primer, $\mathrm{NaOCl}$, and Primer $/ \mathrm{NaOCl}$ against $S$. mutans were determined. The results confirmed that the MIC and MBC values of Primer/ $\mathrm{NaOCl}$ were two times greater than those of Primer only because MDPB in the primer was diluted to half of its concentration due to mixture with $\mathrm{NaOCl}$. Therefore, based on the results of agar disc diffusion tests and measurement of $\mathrm{MIC} / \mathrm{MBC}$ values, the first null hypothesis - the use of $\mathrm{NaOCl}$ provides additional antibacterial effects to Clearfil SE Protect primer-was rejected. However, we considered that remnant chloride ions after $\mathrm{NaOCl}$ application do not obstruct the ability of positively charged MDPB to act on bacteria, even though they do not enhance the antibacterial capacity of the primer.

Several studies reported that $\mathrm{NaOCl}$ treatment before application of self-etch adhesives affects bond strengths of the adhesives. Ozturk and Ozer ${ }^{10)}$ reported that the application of $5 \% \mathrm{NaOCl}$ for 1 min significantly decreased the bond strength of a self-etching adhesive Clearfil SE Bond because $\mathrm{NaOCl}$ removed collagen fibers and consequently prevented the formation of the healthy hybrid layer. In addition, reactive residual free-radicals in $\mathrm{NaOCl}$ may compete with propagating vinyl free-radicals during the light-curing procedure for adhesive systems, which might result in immature and incomplete polymerization ${ }^{23)}$. Conversely, Mohammed et al. ${ }^{8)}$ reported that $4 \% \mathrm{NaOCl}$ application for $20 \mathrm{~s}$ increased the bond strength of a self-etching adhesive Adper Easy One (3 M ESPE, Seefeld, Germany) because the penetration of the primer was increased by removing the collagen layer, smear layer, and smear plug by $\mathrm{NaOCl}$. These studies have shown controversial results of bond strength of self-etching adhesives after the pretreatment of $\mathrm{NaOCl}$.

Our results indicated that pretreatment with 5.25\% $\mathrm{NaOCl}$ for $10 \mathrm{~s}$ did not have a negative influence on the bond strength of Clearfil SE Protect. The disagreement between the results of the present study and those of Ozturk and Ozer $^{10)}$ may be attributed to differences in the processing time of $\mathrm{NaOCl}$. In this study, $5.25 \%$ $\mathrm{NaOCl}$ aqueous solution was used but applied on dentin surface for $10 \mathrm{~s}$, considering the clinical use of $\mathrm{NaOCl}$ as a cavity cleanser. Because the shorter processing time of $\mathrm{NaOCl}$ results in decreased removal of the smear layer and reduced generation of residual free-radicals, $\mathrm{NaOCl}$ treatment may not affect the bonding ability of Clearfil SE Protect.

In attempts to estimate the lifetime of the bond strength at the tooth-composite interface, it became important to obtain fracture mode data. In the primer only (SEP) group, adhesive failure was observed in all specimens. Conversely, pretreatment with $\mathrm{NaOCl}$ before application of the primer $(\mathrm{NaOCl}+\mathrm{SEP})$ demonstrated a lower rate of adhesive failure and cohesive or mixed failure was observed, indicating that bond strength between dentin and the adhesive would not be less than the values obtained. These findings support the greater bonding ability of the $\mathrm{NaOCl}+\mathrm{SEP}$ group to dentin compared with that of the SEP group. Therefore, the second null hypothesis - pretreatment with $\mathrm{NaOCl}$ reduces the bond strength of Clearfil SE Protect- was rejected.

Long-term studies have shown that the bond strength of resin-dentin bonds decreases over time due to collagen degradation within the hybrid layer ${ }^{24,25)}$. However, our group has confirmed that Clearfil SE Protect primer or a cavity disinfectant containing MDPB effectively prevents bond strength reduction after 1-year water storage (unpublished data). It is considered that the bonding durability of the adhesive system can be improved by MDPB, which can inhibit matrix metalloproteases and cathepsins ${ }^{26,27)}$. Further studies should evaluate the influence of pretreatment with $\mathrm{NaOCl}$ on the bonding durability of Clearfil SE Protect.

\section{CONCLUSION}

Employing agar disc diffusion tests and measurement of $\mathrm{MIC} / \mathrm{MBC}$ values, we found that $\mathrm{NaOCl}$ provided no additional effects to the antibacterial activity of Clearfil SE Protect primer containing MDPB. In addition, pretreatment with $5.25 \% \mathrm{NaOCl}$ aqueous solution for $10 \mathrm{~s}$ did not reduce the microtensile bond strength of Clearfil SE Protect to dentin. Within the limitations of this study, it is suggested that enhancement of cavity 
disinfecting effects is not expected by the treatment of $\mathrm{NaOCl}$ before the application of Clearfil SE Protect, although no influence on dentin bonding ability can be obtained.

\section{ACKNOWLEDGMENTS}

This work was supported in part by Grants-in-Aid for Scientific Research (Nos. JP16K20497, JP16K15800, 17K17132, and 17K17128) from the Japan Society for the Promotion of Science.

\section{REFERENCES}

1) Cardoso MV, Neves A, Mine A, Coutinho E, Van Landuyt K, De Munck J, Van Meerbeek B. Current aspects on bonding effectiveness and stability in adhesive dentistry. Aust Dent $J$ 2011; 56: 31-44.

2) Cocco AR, Oliviera de Rosa LW, Da Silva AF, Lund RG, Piva E. A systematic review about antibacterial monomers used in dental adhesive systems: Current status and further perspectives. Dent Mater 2015; 31: 1345-1362.

3) Imazato S, Chen J, Ma S, Izutani N, Li F. Antibacterial resin monomers based on quaternary ammonium and their benefits in restorative dentistry. J Sci Rev 2012; 48: 115-125.

4) Imazato S. Bio-active restorative materials with antibacterial effects: new dimension of innovation in restorative dentistry. Dent Mater J 2009; 28: 11-19.

5) McDonnell G, Russell AD. Antiseptics and disinfectants: activity, action, and resistance. Clin Microbiol Rev 1999; 12: 147-179.

6) Imazato S, Tay FR, Kaneshiro AV, Takahashi Y, Ebisu S. An in vivo evaluation of bonding ability of comprehensive antibacterial adhesive system incorporating MDPB. Dent Mater 2007; 23: 170-176.

7) Bocangel JS, Kraul AOE, Vargas AG, Demarco FF, Matson E. Influence of disinfectant solutions on the tensile bond strength of a fourth generation dentin bonding agents. Pesqiu Odontol Bras 2000; 14: 107-111.

8) Mohammed Hassan A, Ali Goda A, Baroudi K. The effect of different disinfecting agents on bond strength of resin composites. Int J Dent 2014; 2014: 231235.

9) Perdigao J, Lopes M, Gerardeli S, Lopes GS, Garcia-Godoy F. Effect of a sodium hypochlorite gel on dentin bonding. Dent Mater 2000; 16: 311-323

10) Ozturk $\mathrm{B}$, Ozer FE. Effect of $\mathrm{NaOCl}$ on bond strengths of bonding agents to pulp chamber lateral walls. J Endod 2004; 30: 362-365.

11) Hirose N, Kitagawa R, Kitagawa H, Maezono H, Mine A, Hayashi M, Haapasalo M, Imazato S. Development of a cavity disinfectant containing antibacterial monomer MDPB. J Dent Res 2016; 95: 1487-1493.

12) Pascon FM, Kantovitz KR, Sacramento PA, Nobre-dos-Santos M, Puppin-Rontani RM. Effect of sodium hypochlorite on dentine mechanical properties. A review. J Dent 2009; 37: 903-908.

13) Prasansuttiporn T, Nakajima M, Foxton RM, Tagami J. Scrubbing effect of self-etching adhesives on bond strength to $\mathrm{NaOCl}$ treated dentin. J Adhes Dent 2012; 14: 121-127.

14) van Dijken JW. The effect of cavity pretreatment procedures on dentin bonding: a four-year clinical evaluation. J Prosthet Dent 1990; 64: 148-152.

15) Cha HS, Shin DH. Antibacterial capacity of cavity disinfectants against Streptococcus mutans and their effects on shear bond strength of a self-etch adhesive. Dent Mater J 2016; 35: 147-152.

16) Botelho MG. The antimicrobial activity of a dentin conditioner combined with antibacterial agents. Oper Dent 2005; 30: 7582.

17) White RR, Janer LR, Hays GL. Residual antimicrobial activity associated with a chlorhexidine endodontic irrigant used with sodium hypochlorite. Am J Dent 1999; 12: 148-150.

18) Kitagawa H, Izutani N, Kitagawa R, Maezono H, Yamaguchi M, Imazato S. Evolution of resistance to cationic biocides in Streptococcus mutans and Enterococcus faecalis. J Dent 2016; 47: 18-22.

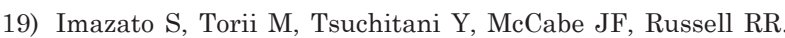
Incorporation of bacterial inhibitor into resin composite. $\mathrm{J}$ Dent Res 1994; 73: 1437-1443.

20) Imazato S, Kinomoto $Y$, Tarumi H, Torii M, Russell RRB, McCabe JF. Incorporation of antibacterial monomer MDPB in dentin primer. J Dent Res 1997; 76: 768-772.

21) Imazato S, Ehara A, Torii M, Ebisu S. Antibacterial activity of dentine primer containing MDPB after curing. J Dent 1998; 26: 267-271.

22) Imazato S, Walls AWG, Kuramoto A, Ebisu S. Penetration of an antibacterial dentine-bonding system into demineralized human root dentine in vitro. Eur J Oral Sci 2002; 110: 168174.

23) Lai SC, Mak YF, Cheung GS, Osorio R, Toledano M, Carvalho RM, Tay FR, Pashley DH. Reversal of compromised bonding to oxidized etched dentin. J Dent Res 2001; 80: 1919-1924.

24) De Munck J, Van Meerbeek B, Yoshida Y, Inoue S, Vargas M, Suzuki K, Lambrechts P, Vanherle G. Four-year water degradation of total-etch adhesives bonded to dentin. J Dent Res 2003; 82: 136-140

25) Koshiro K, Inoue S, Tanaka T, Koase K, Fujita M, Hashimoto $\mathrm{M}$, Sano H. In vivo degradation of resin-dentin bonds produced by a self-etch vs. a total-etch adhesive system. Eur J Oral Sci 2004; 112: 368-375.

26) Tezvergil-Mutluay A, Agee KA, Uchiyama T, Imazato S, Mutluay MM, Cadenaro M, Breschi L, Nishitani Y, Tay FR, Pashley DH. The inhibitory effects of quaternary ammonium methacrylates on soluble and matrix-bound MMPs. J Dent Res 2011; 90: 535-540.

27) Tezvergil-Mutluay A, Agee KA, Mazzoni A, Carvalho RM, Carrilho M, Tersariol IL, Nascimento FD, Imazato S, Tjäderhane L, Breschi L, Tay FR, Pashley DH. Can quaternary ammonium methacrylates inhibit matrix MMPs and cathepsins? Dent Mater 2015; 31: e25-32. 